\title{
PCR based high risk HPV testing is superior to neural network based screening for predicting incident CIN III in women with normal cytology and borderline changes
}

\author{
L Rozendaal, J Westerga, J C van der Linden, J M M Walboomers`, F J Voorhorst, \\ E K J Risse, M E Boon, C J L M Meijer
}

\begin{abstract}
BackgroundlAims-To improve the accuracy of conventional cytology in cervical cancer screening, high risk human papillomavirus (HPV) testing and neural network based screening have been developed. This study assessed the power of both techniques to detect women at risk of developing incident CIN III; that is, CIN III detected during the follow up of women with normal cytology and borderline nu-
\end{abstract} clear changes.

Methods-A cohort of 2250 women, 34-54 years of age, who attended population based cervical cancer screening from 1988 to 1991 and had normal smears or borderline nuclear changes was followed. All smears were tested for high risk HPV and the smears were rescreened using neural network based screening. The value of neural network based screening for predicting incident CIN III during a mean follow up period of 6.4 years was compared with that of high risk HPV testing. In addition, morphological markers presumed to be related to HPV were correlated with HPV status.

Results-Thirteen $(0.6 \%)$ women had incident CIN III. Both high risk HPV positivity and abnormal cytology were associated with an increased risk for incident CIN III (odds ratio, 240 and 22, respectively) and high risk HPV positivity was associated with abnormal cytology. The sensitivity of high risk HPV testing for predicting incident CIN III was much higher than that of neural network based screening (92\% and $46 \%$, respectively). None of the morphological markers assessed, including koilocytosis, was correlated with high risk HPV status.

Conclusion-High risk HPV testing is superior to neural network based screening in identifying women at risk of developing CIN III. For women with normal cytology and borderline changes and a negative high risk HPV test, the screening interval can be considerably prolonged.

(f Clin Pathol 2000;53:606-611)

Keywords: neural network based screening; high risk human papillomavirus testing; CIN III
Classic cervical cytology, introduced several decades ago, is an effective method to reduce the incidence of, and mortality from, cervical cancer. ${ }^{1-6}$ It performs best in the presence of highly abnormal cells indicating more severe abnormalities. However, the limitations of classic cytology have become clear as the prevalence of highly abnormal cervical smears has decreased, partly as the result of the effect of previous screening rounds. Sensitivity values for high grade cervical lesions as high as $85 \%$ and as low as $50 \%$ have been reported, ${ }^{37}$ and specificity does not exceed $90 \%{ }^{8} 9$

Several strategies have been introduced to overcome these limitations of cervical cytology. Some new techniques aim at increasing the accuracy of cytological screening using systems such as neural network based screening, AutoPap 300 QC, AUTOCyt, and Cyto-Savant. ${ }^{10-16}$ Others aim at improvement of the quality of the smears using special preparations, such as ThinPrep and CytoRich. ${ }^{17-21}$ Neural network based screening is used in routine cervical cancer screening ${ }^{10}$ and it is highly sensitive to clinically relevant false negative cervical smears. ${ }^{11}{ }^{12}$

Another approach is based on the demonstration of infection with high risk human papillomavirus (HPV) by means of molecular biological techniques. ${ }^{22-24}$ The development of cervical cancer requires a preceding and persisting infection with this virus. ${ }^{25-34}$ Rozendaal and colleagues ${ }^{32}$ have shown that, among 1622 women with normal cytology in population based cervical cancer screening, the presence of high risk HPV was associated with an increased risk of developing CIN III during a follow up period of 4.5 years (odds ratio (OR), 116). The risk of developing CIN III in the case of a negative HPV test result was so low that a prolonged screening interval was proposed for women with normal cytology, borderline nuclear changes, and a negative high risk HPV test. In our study, we extended these observations and compared the performance of high risk HPV testing and neural network based screening for predicting incident CIN III.

\section{Material and methods}

STUDY GROUP

During 1988 to 1991,4079 women, who attended the Dutch population based cervical cancer screening programme, participated in a study on the age related prevalence of high risk HPV in cervical smears. ${ }^{35}$ In 1999, the pathol-
Accepted for publication 7 January 2000 
Table 1 High risk human papillomavirus (HPV) testing, neural network based screening (NNS), and prediction of CIN III

\begin{tabular}{llll}
\hline Subgroup of women & $\begin{array}{l}\text { Number of } \\
\text { women }\end{array}$ & $\begin{array}{l}\text { Mean age in years } \\
\text { (range) }\end{array}$ & $\begin{array}{l}\text { Mean follow up time } \\
\text { in years (range) }\end{array}$ \\
\hline $\begin{array}{l}\text { Group A analysis } \\
\text { All women in this analysis }\end{array}$ & 2250 & $43.0(34-54)$ & $6.4(0.5-10.2)$ \\
Incident CIN III & 13 & $38.6(34-53)$ & $3.9(0.9-9.8)$ \\
No CIN III & 2237 & $43.0(34-54)$ & $6.4(0.5-10.2)$ \\
High risk HPV positive & 121 & $43.0(34-54)$ & $6.1(0.9-9.8)$ \\
High risk HPV negative & 2129 & $43.0(34-54)$ & $6.4(0.5-10.2)$ \\
Group B analysis & & & \\
All women in this analysis & 653 & $42.7(34-54)$ & $6.2(0.5-10.1)$ \\
Incident CIN III & 13 & $38.6(34-53)$ & $3.9(0.9-9.8)$ \\
No CIN III & 640 & $42.8(34-54)$ & $6.2(0.5-10.1)$ \\
NNS abnormal ( $\geqslant$ Pap 2) & 30 & $43.0(34-53)$ & $6.2(0.9-9.1)$ \\
NNS normal (Pap 1) & 623 & $42.7(34-54)$ & $6.2(0.5-10.1)$ \\
Group C analysis & 731 & $42.7(34-54)$ & $6.2(0.5-10.1)$ \\
All women in this analysis & 38 & $43.2(34-53)$ & $6.2(0.9-9.1)$ \\
NNS abnormal ( Pap 2) & 693 & $42.7(34-54)$ & $6.2(0.5-10.1)$ \\
NNS normal (Pap 1) & 121 & $43.0(34-53)$ & $6.1(0.9-9.8)$ \\
High risk HPV positive & 610 & $42.7(34-54)$ & $6.2(0.5-10.1)$ \\
High risk HPV negative & &
\end{tabular}

Group A analysis: women included in the analysis of high risk HPV testing and predicting incident CIN III.

Group B analysis: women included in the analysis of NNS and predicting incident CIN III. Group C analysis: women included in the analysis of the association between high risk HPV status and NNS result.

Women with incident CIN III are younger and have a shorter follow up time when compared with women without CIN III. This is an effect of screening. The follow up time is shorter because 8/13 women with incident CIN III were diagnosed within three years of follow up. They are younger because only very few of the older women with a normal smear or borderline nuclear changes in two or more previous screening rounds will still develop CIN III.

ogy report database of the academic hospital Vrije Universiteit and the Dutch national pathology report database (PALGA) were searched for follow up data of this cohort.

Inclusion criteria were: no history of previous cervical abnormalities at baseline, a normal cervical smear or borderline nuclear changes in the original routine screening, a valid high risk HPV test result, and availability of follow up cervical smears or biopsies. A total of 2250 women met these criteria and were included in the analysis. Table 1 presents the epidemiological data of this group and the various subgroups defined in our study (vide infra).

\section{INCIDENT CIN III}

Incident CIN III was used as the endpoint of the study. CIN III is generally considered to be the most advanced premalignant stage before invasive carcinoma. Because all women had normal cytology or borderline nuclear changes at baseline, they were considered not to have CIN III at baseline. Incident CIN III was detected and histologically confirmed as a result of abnormal cytology in successive screening rounds, typically every three years till 1995 and every five years since 1996. According to the Dutch referral policy at that time, women were referred to the gynaecologist for colposcopically directed biopsy sampling and treatment after a single smear assessed as severe dyskaryosis or worse, or two smears assessed as mild to moderate dyskaryosis in an interval of three months. Women with a smear showing borderline nuclear changes were advised to have the smear repeated after a period of one year.

SAMPLING FOR CERVICAL CYTOLOGY AND HIGH RISK HPV TESTING

Scrapes from the cervix uteri were obtained by the general practitioner by means of a cervex brush (International Medical Products, Zut- phen, the Netherlands). A conventional smear was made on a glass slide and the remaining cell material on the brush was used for the detection of 14 high risk HPV types $(16,18$, $31,33,35,39,45,51,52,56,58,59,66$, and 68 ) in a single test by GP5+/6+ polymerase chain reaction enzyme immune assay (PCREIA), as described previously. ${ }^{32} 36$ The laboratory staff were unaware of the Papanicolaou (Pap) smear result and follow up data.

\section{CLASSIFICATION OF CERVICAL SMEARS}

For cytological review of the smears, neural network based screening was used. For neural network based screening, we used a PAPNET device. ${ }^{11}{ }^{12}$ In short, cytological images were digitised and the most suspicious parts were automatically selected. These automatically selected parts were judged by cytotechnologists. Smears suspected of abnormal cells were evaluated light microscopically by an expert pathologist, who rendered the final diagnosis. Both cytotechnologists and pathologists were unaware of the high risk HPV status and follow up data.

To simulate routine cervical cancer screening to the cytotechnologists, which would have a certain number of abnormal cervical smears, 31 abnormal smears $(4.1 \%)$ were added (14 with Pap 3a, 12 Pap 3b, four Pap 4, and one Pap 5). These smears were derived from population based cervical cancer screening in the same period as the smears included in the analysis. The cytotechnologists could not recognise these smears from their labelling, colouring, or any other properties. Because these smears were only added to simulate a routine screening setting and keep the cytotechnologists alert, they were excluded from analysis.

The smears were cytomorphologically assessed according to the standardised Dutch KOPAC-B coding system, modified after Vooijs et al. ${ }^{38}$ The English abbreviation of KOPAC-B is CISOC-A: composition, inflammation, squamous epithelium, other abnormalities, and endometrium, columnar epithelium, and adequacy of the smear. In the coding system, each of the five CISOC values is assigned a value between 0 and 9. Only the SOC values affect the Pap classification. In short, Pap 0 indicates an inadequate smear; Pap 1 a normal smear; Pap 2 borderline nuclear changes, including atypical squamous cells of unknown significance (ASCUS); Pap 3a1 mild dyskaryosis; Pap 3a2 moderate dyskaryosis; Pap 3b severe dyskaryosis; Pap 4 carcinoma in situ; and Pap 5 invasive carcinoma.

ANALYSIS OF HPV AND NEURAL NETWORK BASED SCREENING DATA

The analyses focused on four issues.

High risk HPV testing and predicting incident CIN III

For high risk HPV testing and predicting incident CIN III, the data of all 2250 women were used in a cohort study design (table 1; group A). 
Table 2 Characteristics at baseline of the 13 women with incident CIN III

\begin{tabular}{lllllll}
\hline Case & Age (years) & $\begin{array}{l}\text { Routine } \\
\text { screening (Pap) }\end{array}$ & NNS (Pap) & $\begin{array}{l}\text { High risk } \\
\text { HPV }\end{array}$ & $\begin{array}{l}\text { Follow up } \\
\text { time (years) }\end{array}$ & CIN \\
\hline 1 & 34 & 1 & 1 & Yes & 2.1 & 3 \\
2 & 35 & 2 & $3 \mathrm{a} 2$ & Yes & 2.6 & 3 \\
3 & 35 & 1 & 1 & Yes & 3.4 & 3 \\
4 & 35 & 2 & 2 & Yes & 2.6 & 3 \\
5 & 35 & 1 & 1 & Yes & 5.7 & 3 \\
6 & 36 & 1 & 1 & Yes & 2.9 & 3 \\
7 & 38 & 1 & $3 \mathrm{a} 2$ & Yes & 3.0 & 3 \\
8 & 38 & 1 & 2 & Yes & 1.5 & 3 \\
9 & 38 & 1 & 1 & Yes & 7.9 & 3 \\
10 & 42 & 1 & 1 & Yes & 5.1 & 3 \\
11 & 42 & 1 & 2 & Yes & 8.9 & 3 \\
12 & 53 & 2 & $3 \mathrm{a} 1$ & Yes & 0.9 & 3 \\
13 & 41 & 2 & 1 & No* & 4.7 & 3 \\
\hline
\end{tabular}

The 13 women listed in this table had histologically confirmed CIN III.

* This woman acquired high risk HPV after about two years and incident CIN III was found after another three years of follow up.

HPV, human papillomavirus; NNS, neural network based screening.

Table 3 Baseline high risk HPV status and incident CIN III during follow up

\begin{tabular}{llll}
\hline & \multicolumn{2}{l}{ Incident CIN III } & \\
\cline { 2 - 3 } Baseline high risk HPV status & Yes & No & Total \\
\hline High risk HPV positive & 12 & 109 & 121 \\
High risk HPV negative & $1^{\star}$ & 2128 & 2129 \\
Total & 13 & 2237 & 2250 \\
\hline
\end{tabular}

*This woman acquired high risk HPV after about two years and incident CIN III was found after another three years of follow up.

HPV, human papillomavirus.

Table 4 Baseline NNS result and incident CIN III during follow up

\begin{tabular}{llll}
\hline \multirow{2}{*}{ NNS result } & \multicolumn{2}{l}{ Incident CIN III } & \multirow{2}{*}{ Total } \\
\cline { 2 - 3 } & Yes & No & \\
\hline Abnormal ( $\geqslant$ Pap 2) & 6 & 24 & 30 \\
Normal (Pap 1) & 7 & 616 & 623 \\
Total & 13 & 640 & 653 \\
\hline
\end{tabular}

The smears of all 13 women with incident CIN III have been included in this nested case control analysis. Of the 2237 women who did not develop CIN III 640 smears have been reviewed.

HPV, human papillomavirus; NNS, neural network based screening.

Neural network based screening and predicting incident CIN III

The value of neural network based screening for predicting incident CIN III was assessed in a nested case control study design. The smears from the women with incident CIN III (13 patients) and a random selection of the smears from the 2237 women without incident CIN III (640 controls) were reviewed (table 1; group B).

\section{Association between high risk HPV status and} neural network based screening result

The association between high risk HPV and neural network based screening result was analysed in a subgroup of women comprising all women with a positive high risk HPV test and a random selection of the women with a negative high risk HPV test (table 1; group $\mathrm{C}$ ).

Reliability of determination of HPV status on the basis of morphological criteria

Specific morphological cytonuclear characteristics have been related to the presence of HPV - for example, koilocytosis, parakeratosis, and multinucleation. ${ }^{39-41}$ The relation between these morphological signs with the presence of high risk HPV was studied.

\section{STATISTICAL METHODS}

Logistic regression and $\mathrm{McNemar}$ analysis were used where appropriate. Sensitivity, specificity, positive and negative predictive values, and relative risk were computed using two by two tables. Because the value of neural network based screening was assessed in a case control study design, the positive and negative predictive values could not be computed directly. To estimate these values of neural network based screening, an estimate of the number of abnormal smears among all 2250 women was needed. To this end, the neural network based screening results of the 640 women without CIN III tested (the 640 controls) were extrapolated to all 2237 women without CIN III.

\section{Results}

During the follow up period (mean, 6.4 years; range, $0.5-10.2), 13$ of $2250(0.6 \%)$ women developed histologically confirmed CIN III. Table 2 summarises the main characteristics of these 13 women, together with the original screening result, HPV status, and neural network based screening result at baseline. Of the women with incident CIN III, 12 had a positive high risk HPV test at baseline. The woman with incident CIN III and a negative high risk HPV test at baseline acquired high risk HPV after about two years of follow up and CIN III was diagnosed after another three years of follow up. Thus, in all women with incident CIN III an infection with high risk $\mathrm{HPV}$ was present before the diagnosis of CIN III.

\section{HIGH RISK HPV TESTING AND PREDICTING}

INCIDENT CIN III

In this analysis, the data from all 2250 women were used (table 1; group A). At baseline, high risk HPV was present in 121 of $2250(5.4 \%)$ women, and $12(10 \%)$ of them developed CIN III, whereas only one $(0.05 \%)$ of the 2129 women with a negative high risk HPV test at baseline developed CIN III (table 3; relative risk, 210; 95\% confidence interval (CI), 27 to 1600).

The number of women with incident CIN III can also be expressed for each 1000 women years of follow up. This value was 16.2 among women with a positive high risk HPV test at baseline and 0.073 in the case of a negative high risk HPV test result.

NEURAL NETWORK BASED SCREENING AND PREDICTING INCIDENT CIN III

Among the 2250 women included in the analysis of high risk HPV testing, a nested case control study was performed (table 1; group B). In this analysis, 653 smears were reviewed, consisting of 13 patients (all women with incident CIN III) and 640 controls (randomly drawn from the 2237 women without CIN III). Of these 653 smears, 30 were abnormal: 26 Pap 2, two Pap 3a1, and two Pap 3a2. Of the 30 
Table 5 Logistic regression analysis for incident CIN III

\begin{tabular}{lllll}
\hline Baseline risk factor & $\begin{array}{l}\text { Cases } \\
(n=13)\end{array}$ & $\begin{array}{l}\text { Controls } \\
(n=640)\end{array}$ & $\begin{array}{l}\text { Unadjusted } \\
\text { OR }\end{array}$ & $\begin{array}{l}\text { Mutually adjusted } \\
\text { OR (95\% CI })\end{array}$ \\
\hline $\begin{array}{l}\text { Positive high risk HPV test } \\
\text { NNS abnormal ( Pap 2) }\end{array}$ & 6 & 31 & 240 & $\begin{array}{l}160(20 \text { to 1300) } \\
4.8(1.2 \text { to 20) }\end{array}$ \\
\hline
\end{tabular}

CI, confidence interval; HPV, human papillomavirus; NNS, neural network based screening; OR, odds ratio.

Table 6 Comparison of test parameters of high risk HPV and NNS review for incident CIN III

\begin{tabular}{llllll}
\hline & \multicolumn{2}{l}{ High risk } & HPV test & & \multicolumn{2}{c}{ NNS review } \\
\cline { 2 - 3 } \cline { 5 - 6 } & $\%$ & $n$ & & $\%$ & $n$ \\
\hline Sensitivity & 92 & $12 / 13$ & & 46 & $6 / 13$ \\
Positive predictive value & 9.9 & $12 / 121$ & & 6.7 & $6 / 90$ \\
Specificity & 95 & $2128 / 2237$ & & 96 & $2153 / 2237$ \\
Negative predictive value & 99.9 & $2128 / 2129$ & & 99.7 & $2153 / 2160$ \\
\hline
\end{tabular}

In this table, the figures concerning high risk HPV testing are directly copied from the cohort analysis presented in table 3 . The figures concerning NNS review are derived from the nested case control analysis presented in table 4 by multiplying the results of the women who did not develop CIN III by 2237/640.

HPV, human papillomavirus; NNS, neural network based screening.

Table 7 NNS result and high risk HPV status at baseline

\begin{tabular}{llllll}
\hline \multirow{5}{*}{$\begin{array}{l}\text { High risk HPV } \\
\text { status }\end{array}$} & \begin{tabular}{l} 
Pap \\
\cline { 2 - 5 }
\end{tabular} & $\begin{array}{l}\text { Pap } \\
\text { 3a1 }\end{array}$ & Pap 2 & Pap 1 & Total \\
\hline Positive & 2 & 2 & 15 & 102 & 121 \\
Negative & 2 & 1 & 18 & 591 & 610 \\
Total & 2 & 33 & 693 & 731 \\
\hline
\end{tabular}

HPV, human papillomavirus; NNS, neural network based screening.

women with abnormal cytology, six developed CIN III, whereas only seven of 623 women with normal cytology did so (table 4; OR, 22; $95 \%$ CI, 6.9 to 70$)$.

A logistic regression model, which mutually adjusted the risks for incident CIN III associated with high risk HPV and abnormal cytology, showed a significant independent risk of abnormal cytology (table 5; OR, 4.8; $95 \%$ CI, 1.2 to 20 ).

With regard to the grading of abnormal cytology at baseline, of the 13 women with incident CIN III, six showed abnormal cytology: three Pap 2, one Pap 3a1, and two Pap 3a2 (table 2). Three of the 13 women with incident CIN III had also been signalled by the original screening (Pap 2). Of the 640 women without CIN III, 24 showed abnormal cytology: 23 Pap 2, and one Pap 3a1.

With regard to high risk HPV and neural network based screening testing, the parameters of sensitivity, specificity, and positive and negative predictive value are of interest. For

Table 8 Morphological markers associated with HPV v high risk HPV test result

\begin{tabular}{lllll}
\hline & \multicolumn{3}{l}{ High risk HPV } \\
\cline { 2 - 5 } Morphological marker present & $\begin{array}{l}\text { Positive } \\
\left(\begin{array}{l}n=121) \\
\%\end{array}\right.\end{array}$ & $\begin{array}{l}\text { Negative }(n= \\
610) \\
n\end{array}$ & $\%$ \\
\hline Nuclear and cytoplasmatic enlargement & 4 & 3.3 & 22 & 3.6 \\
Multinucleation & 5 & 4.0 & 41 & 6.7 \\
Koilocytosis & 5 & 4.0 & 21 & 3.4 \\
Parakeratosis & 4 & 3.3 & 26 & 4.3 \\
Mildly) hyperchromatic nucleus & 1 & 0.8 & 3 & 0.5 \\
Atypical reserve cell hyperplasia & 4 & 3.3 & 6 & 1.0 \\
Immature squamous metaplasia & 1 & 1.7 & 14 & 2.3 \\
Any marker present & 14 & 12 & 79 & 13 \\
\hline
\end{tabular}

HPV, human papillomavirus. high risk HPV, these parameters can be derived from the data presented in table 3. For neural network based screening, the results of the 640 controls as presented in table 4 have to be used to estimate the number of women with abnormal cytology among all 2237 women without CIN III. To this end, the neural network based screening results of the 640 controls were multiplied by $2237 / 640$ (the reciprocal of the sampling fraction). Table 6 presents the computed test properties for high risk HPV testing and neural network based screening. High risk HPV testing is twice as sensitive as neural network based screening at detecting women at risk of developing CIN III (92\% and 46\%, respectively). This difference is significant (McNemar, $\left.\chi^{2}=4.2 ; \mathrm{p}=0.04\right)$. For both tests, the positive predictive values are rather low (9.9\% and $6.7 \%$, respectively); the specificity (95\% and $96 \%$, respectively) and negative predictive values (99.9\% and $99.7 \%$, respectively) are similar.

ASSOCIATION BETWEEN HIGH RISK HPV STATUS AND NEURAL NETWORK BASED SCREENING RESULT

For this subgroup analysis, a total of 731 smears were selected (table 1; group C). These comprised the smears of all 121 women with a positive high risk HPV test at baseline and 610 smears randomly drawn from the 2129 women with a negative high risk HPV test (table 7). In 19 of $121(16 \%)$ women with a positive high risk HPV test, the Pap smear was abnormal. A much smaller proportion (19 of $610(3.1 \%)$ ) of the women with a negative high risk HPV test had abnormal cytology. Thus, abnormal cytology is correlated with a positive high risk HPV test (OR, 5.8; 95\% CI, 3.0 to 11$)$.

RELIABILITY OF DETERMINATION OF HPV STATUS ON THE BASIS OF MORPHOLOGICAL CRITERIA

Table 8 lists the results of the seven markers assessed. None of these markers was significantly correlated with a positive high risk HPV test.

\section{Discussion}

The main finding of our study was that the sensitivity of high risk HPV testing in predicting incident CIN III is twice as high as the sensitivity of neural network based screening (92\% and $46 \%$, respectively). Abnormal cytology was correlated with a positive high risk HPV test. None of seven markers (including koilocytosis) presumed to be associated with an HPV infection were significantly associated with a positive GP5+/6+ PCR-EIA high risk HPV test.

Previously, it has been shown that high risk HPV testing could identify those women with normal cytology who are at risk of developing CIN III. ${ }^{25}{ }^{32}$ In these studies, conventional cervical cytology had been used. It has become clear that the accuracy of conventional cytology can be increased by using computer assisted screening - for example, neural network based screening. ${ }^{10-12}$ The improved accuracy of neural network based screening when compared with conventional screening has led to the question 
of whether neural network based screening, like high risk HPV testing, could identify women at risk of developing CIN III. To answer this question, we compared neural network based screening with high risk HPV testing. As stated above, neural network based screening identified six of $13(46 \%)$ women with incident CIN III, whereas high risk HPV testing identified 12 of $13(92 \%)$ women. This difference was significant. We conclude therefore that high risk HPV testing is superior to neural network based screening in identifying women at risk of incident CIN III. The better performance of high risk HPV testing, when compared with neural network based screening, can be explained by its ability to identify those women with normal cytology who are at risk of developing CIN III.

Incident CIN III was always confirmed histologically. On the one hand, the estimated number of women with incident CIN III might have been too high: at baseline, CIN status had not been verified histologically and some of the women with incident CIN III might have had CIN III from the outset of the study. On the other hand, the estimated number of women with incident CIN III might have been too low: not all women with incident CIN III might have been detected by cytology, some cases of incident CIN III might have stayed undetected. It is probable that these contrary effects on incident CIN III will have balanced out and, taking both effects into account, would not have affected the overall results.

Some morphological markers, including koilocytosis, have been associated with HPV infections. ${ }^{39}$ We looked for a relation between these markers and the presence of high risk $\mathrm{HPV}$, because only high risk HPV is associated with progression to CIN III, among women with both normal and abnormal cytology. ${ }^{25} 27303233$ We did not find a correlation between the presence of high risk HPV and any of the seven markers assessed (table 8). As a consequence, the markers assessed were not associated with incident CIN III. None of markers was present in the seven women with normal cytology who developed incident CIN III. Thus, assessment of these markers cannot substitute for high risk HPV testing by molecular biological techniques. Our results are in contrast with those of Cramer et $a l,{ }^{40}$ who found a positive correlation between koilocytosis and high risk HPV, as detected by the hybrid capture assay, in a matched case control study among 126 young women (age, 18-45 years) attending a gynaecological outpatient clinic or a sexually transmitted disease clinic. However, Abadi and colleagues ${ }^{41}$ only found a weak positive correlation between koilocytosis and high risk HPV, as detected by MY09/11 PCR, when stringent criteria were used for koilocytosis. No correlation had been present between a positive high risk HPV test and koilocytosis in a routine diagnostic setting.

The specificity of high risk HPV testing and neural network based screening are similar ( $95 \%$ and $96 \%$, respectively). In population based cervical cancer screening, these specificity values are too low to justify referral for col- poscopy after a single positive test. Therefore, women with borderline nuclear changes are not referred for colposcopy, unless at a six months interval a repeated smear again shows borderline nuclear changes or worse. ${ }^{8}$ For high risk HPV testing we also recommend a repeated high risk HPV test because only persisting HPV infections eventually lead to high grade CIN lesions. ${ }^{30}{ }^{33}$ Women with a negative second high risk HPV test and normal or borderline cytology should not be referred. With respect to repeated tests, Nobbenhuis and colleagues ${ }^{33}$ have shown that a second high risk HPV test performs better than repeated cytology in identifying women at risk of CIN III.

One of the major concerns in cervical cytology is quality assurance. ${ }^{39}$ In agreement with the observation of Sherman, ${ }^{9}$ we found that abnormal cytology is correlated with a positive high risk HPV test (table $7^{7}$; OR, 5.8; 95\% CI, 3.0 to 11$)$. Therefore, high risk HPV testing can serve as a quality control of cytology. The number of false negative smears can be substantially reduced (in our study by $50 \%$ ) by selectively reviewing high risk HPV positive smears.

Women with abnormal cytology had an increased risk for incident CIN III (table 4). The strong correlation between an infection with high risk HPV and abnormal cytology indicates that the cellular changes are largely induced by high risk HPV. The increased risk for incident CIN III among women with abnormal cytology can therefore be attributed almost completely to high risk HPV (table 5). The small independent risk of abnormal cytology for incident CIN III (OR, 4.8; 95\% CI, 1.2 to 20) can be explained using the model of cervical carcinogenesis. ${ }^{42}$ According to this model, most HPV infections are cleared but some persist and may eventually lead to cellular transformation. Abnormal cytology indicates that progression of cellular transformation has occurred to the level of morphological detection. These patients will be more likely to proceed to CIN III than women with an HPV infection but normal cytology.

In summary, the presence of high risk HPV is the best indicator for incident CIN III among women with normal cytology or borderline nuclear changes. Nobbenhuis and colleagues ${ }^{33}$ and $\mathrm{Ho}$ and colleagues ${ }^{27}$ have shown that this also holds for women with mild dyskaryosis or worse. We advocate therefore that both high risk HPV testing and cytology should be used in cervical cancer screening, and we are currently conducting an implementation study of high risk HPV testing in population based screening involving 44000 women in the Netherlands.

We acknowledge Mrs N Fransen Daalmeijer, Mr R Pol, Mr B Bakker, and the cytotechnologists of the LCPL laboratory for technical assistance. We thank the general practitioners in the region of Amstelveen/Ouder-Amstel for their kind cooperation. This work was supported by grants from the Ziekenfondsraad and the Praeventiefonds (project 28-1502).

1 Boyes DA, Morrison B, Knox EG, et al. A cohort study of cervical cancer screening in British Columbia. Clin Invest Med 1982;5:1-29.

2 IARC Working Group on Evaluation of Cervical Cancer Screening Programmes. Screening for squamous cervical 
cancer: duration of low risk after negative results of cervical cytology and its implication for screening policies. BMF

3 Koss LG. The Papanicolaou test for cervical-cancer detection: a triumph and a tragedy. $\mathscr{f} A M A 1989 ; 261: 737-$ 43.

4 Gustafsson L, Adami HO. Natural history of cervical neoplasia: consistent results obtained by an identification technique. Br F Cancer 1989;60:132-41.

5 Van Ballegooijen M, Habbema JDF, van Oortmarssen GJ et al. Preventive Pap-smears: balancing costs, risks and benefits. Br F Cancer 1992;65:930-3.

6 Cannistra SA, Niloff JM. Cancer of the uterine cervix. $N$ Engl F Med 1996;334:1030-8.

7 Van der Graaf Y, Vooijs GP, Gaillaard HLJ, et al. Screening errors in $c$

8 Kurman RJ, Henson D, Herbst A, et al. Interim guidelines for management of abnormal cervical cytology. $7 A M A$ for management

9 Sherman ME, Schiffman MH, Lörincz AT, et al. Toward objective quality assurance in cervical pathology; correla-
tion of cytopathologic diagnoses with detection of high-risk tion of cytopathologic diagnoses with detection of high-risk human $182-7$.

10 Kok MR, Boon ME. Consequences of neural network technology for cervical screening: increase in diagnostic consistency and positive scores. Cancer 1996;78:112-17.

11 Rosenthal DL, Acosta D, Peters RK. Computer-assisted rescreening of clinically important false negative cervical smears using the PAPNET testing system. Acta Cytol 1996; 40:120-6.

12 Doornewaard H, Van de Seijp H, Woudt JMC, et al. Negative cervical smears before CIN 3/carcinoma. Reevaluation with the PAPNET testing system. Acta Cytol 1997;41:74-8.

13 Patten SF, Lee JSJ, Nelson AC. NeoPath, Inc. NeoPath AutoPap 300 automatic Pap screener system. Acta Cytol 1996;40:45-52.

14 Knesel EA. Roche Image Analysis Systems, Inc. Acta Cytol 1996;40:60-6.

15 Palcic B, Garner DM, MacAuley CE, et al. Oncometrics Imaging Corporation and Xillix Technologies Corporation. Use of the Cyto-Savant in quantitative cytology. Acta Cytol 1996;40:67-72.

16 Wilbur DC, Bonfiglio TA, Rutkowski M-A, et al. Sensitivity of the AutoPap 300 QC system for cervical cytologic abnormalities. Acta Cytol 1996;40:127-32.

17 Vassilakos P, Cossali D, Albe X, et al. Efficacy of monolayer preparations for cervical cytology: emphasis on suboptimal specimens. Acta Cytol 1996;40:496-500.

18 Linder J, Zahniser D. The ThinPrep Pap test. A review of clinical studies. Acta Cytol 1997;41:30-8.

19 Laverty CR, Farnsworth A, Thurloe JK, et al. Evaluation of the CytoRich slide preparation process. Anal Quant Cytol Histol 1997:19:239-45.

20 Laverty CR, Thurloe JK, Redman NL, et al. An Australian trial of ThinPrep: a new cytopreparatory technique. trial of ThinPrep: a new

21 Wilbur DC, Facik MS, Rutkowski M-A, et al. Clinical trials of the CytoRich specimen-preparation device for cervical
cytology. Preliminary results. Acta Cytol 1997;41:24-9.

22 Meijer CJLM, Van den Brule AJC, Snijders PJF, et al. Detection of human papillomavirus in cervical scrapes by the polymerase chain reaction in relation to cytology: possible implications for cervical cancer screening. In: Muñoz $\mathrm{N}$, Bosch FX, Shah KV, et al, eds. The epidemiology of human papillomavirus and cervical cancer. Lyon, France: IARC Scientific Publication, 119, 1992:271-81.

23 Cuzick J, Szarewski A, Terry G, et al. Human papillomavirus testing in primary cervical screening. Lancet 1995;345: 1533-6.

24 Jenkins D, Sherlaw-Johnson C, Gallivan S. Can papillomavirus testing be used to improve cervical cancer screening? Int f Cancer 1996;65:768-73.
25 Koutsky LA, Holmes KK, Critchlow CW, et al. A cohort study of the risk of cervical intraepithelial neoplasia grade 2 or 3 in relation to papillomavirus infection. $N$ Engl $f$ Med 992;327:1272-8.

26 Bosch FX, Manos MM, Muñoz N, et al. Prevalence of human papillomavirus in cervical cancer: a worldwide perspective. F Natl Cancer Inst 1995;87:796-802.

27 Ho GYF, Burk RD, Klein S, et al. Persistent genital human papillomavirus infection as a risk factor for persistent cervical dysplasia. F Natl Cancer Inst 1995;87:1365-71.

28 IARC monographs on the evaluation of carcinogenic risks to humans. IARC working group human papillomaviruses. Lyon, France: IARC Scientific Publication, 64, 1995.

29 Schiffman MH, Brinton LA. The epidemiology of cervical carcinogenesis. Cancer 1995;76:1888-901.

30 Remmink AJ, Walboomers JMM, Helmerhorst ThJM, et al. The presence of persistent high-risk HPV genotypes in dysplastic cervical lesions is associated with progressive disease: natural history up to 36 months. Int F Cancer 1995; 61:306-11.

31 Kjær SK, Van den Brule AJC, Bock JE, et al. Human papillomavirus - the most significant risk determinant of cervical intraepithelial neoplasia. Int f Cancer 1996;65: 601-6.

32 Rozendaal L, Walboomers JMM, Van der Linden JC, et al. PCR-based high-risk HPV test in cervical cancer screening gives objective risk assessment of women with cytomorphologically normal cervical smears. Int f Cancer 1996;68:7669.

33 Nobbenhuis MAE, Walboomers JMM, Helmerhorst ThJM, et al. Relation of human papillomavirus status to cervical lesions and consequences for cervical-cancer screening: a prospective study. Lancet 1999;354:20-5.

34 Walboomers JMM, Jacobs MV, Manos MM, et al. Human papillomavirus is a necessary cause of invasive cervical cancer worldwide. F Pathol 1999;189:12-9.

35 Melkert PWJ, Hopman EH, Van den Brule AJC, et al. Prevalence of HPV in cytomorphologically normal cervical smears, as determined by the polymerase chain reaction, is age-dependent. Int 7 Cancer 1993;53:919-23.

36 De Roda Husman AM, Walboomers JMM, Van den Brule AJC, et al. The use of general primers GP5 and GP6 elongated at their 3' ends with adjacent highly conserved sequences improves human papillomavirus detection by PCR. F Gen Virol 1995;76:1057-62.

37 Jacobs MV, De Roda Husman AM, Van den Brule AJC, et al. Group-specific differentiation between high- and low-risk human-papillomavirus genotypes by general primer-mediated PCR and two cocktails of oligonucleotide probes. 7 Clin Microbiol 1995;33:901-5.

38 Vooijs GP. De advisering bij afwijkende bevindingen van cytologisch onderzoek van de cervix uteri. Ned Tijdschr Geneeskd 1987:131:1662-3.

39 Tanaka H, Chua KL, Lindh E, et al. Patients with various types of human papillomavirus: covariation and diagnostic relevance of cytological findings in Papanicolaou smears. Cytopathology 1993;4:273-83.

40 Cramer HM, Skinner-Wannemuehler SE, Brown DR, et al. Cytomorphologic correlates of human papillomavirus infection in the "normal" cervicovaginal smear. Acta Cytol 1997;41:261-8.

41 Abadi MA, Ho GYF, Burk RD, et al. Stringent criteria for histological diagnosis of koilocytosis fail to eliminate overdiagnosis of human papillomavirus infection and cervical intraepithelial neoplasia grade 1. Hum Pathol 1998;29:549.

42 Meijer CJLM, Rozendaal L, Van der Linden JC, et al. Human papillomavirus testing for primary cervical cancer screening. In: Franco E, Monsenego J, eds. New developments in cervical cancer screening 